\title{
Decreased Defibrillation Threshold and Minimized Myocardial Damage With Left Axilla Implantable Cardioverter Defibrillator Implantation
}

\author{
Mahito Noro, MD, PhD; Xin Zhu, PhD; Yoshinari Enomoto, MD; Yasuhiro Oikawa; \\ Hiroyuki Tatsunami, BSc; Rina Ishii, MD; Yasutake Toyoda, MD; Masako Asami, MD;
}

Naohiko Sahara, MD; Takahito Takagi, MD; Yuriko Narabayashi, MD; Hikari Hashimoto, MD;

Naoshi Ito, MD; Shingo Kujime, MD; Tsuyoshi Sakai, MD, PhD; Keijirou Nakamura, MD, PhD; Takao Sakata, MD, PhD; Haruhiko Abe, MD, PhD; Kaoru Sugi, MD, PhD

\begin{abstract}
Background: To reduce myocardial damage caused by implantable cardioverter defibrillator (ICD) shock, the left axilla was studied as an alternative pulse generator implantation site, and compared with the traditional implantation site, the left anterior chest.
\end{abstract}

Methods and Results: Computer simulation was used to study the defibrillation conduction pattern and estimate the simulated defibrillation threshold (DFT) and myocardial damage when pulse generators were placed in the left axilla and left anterior chest, respectively; pulse generators were also newly implanted in the left axilla $(n=30)$ and anterior chest $(n=40)$ to compare the corresponding DFT. On simulation, when ICD generators were implanted in the left axilla, compared with the left anterior chest, the whole heart may be defibrillated with a lower defibrillation energy (left axilla $6.4 \mathrm{~J}$ vs. left anterior chest $12.0 \mathrm{~J}$ ) and thus the proportion of cardiac myocardial damage may be reduced (2.1 vs. $4.2 \%$ ). Clinically, ventricular fibrillation was successfully terminated with a defibrillation output $\leq 5 \mathrm{~J}$ in $86.7 \%(26 / 30)$ of the left axillary group, and in $27.5 \%(11 / 40)$ of the left anterior group $(\mathrm{P}<0.001)$.

Conclusions: Clinically and theoretically, the left axilla was shown to be an improved ICD implantation site that may reduce DFT and lessen myocardial damage due to shock. Lower DFT also facilitates less myocardial damage, as a result of the lower shock required. (Circ J 2016; 80: 878-886)

Key Words: Computer simulation; Defibrillation threshold; Implantable cardioverter defibrillator; Left axilla; Myocardial damage

$\mathbf{I}$ mplantable cardioverter defibrillators (ICD) are widely used for the prevention of sudden cardiac death due to ventricular arrhythmia.1,2 A worsened prognosis has been reported, however, even after appropriate ICD shock, accompanied by a significant increase in the risk of mortality. ${ }^{3,4}$ Further studies have been done to determine whether the adverse prognosis is caused by underlying cardiac arrhythmia or the sideeffect of shocks. ${ }^{5-8}$ Although it is controversial whether the myocardial damage caused by ICD shock worsens the prognosis, ${ }^{3}$ previous studies indicated that ICD shock causes myo- cardial damage, and severity is related to the defibrillation energy. ${ }^{9-13}$

\section{Editorial p 816}

Although the outcome of myocardial damage is controversial regarding the prognosis, it is hypothesized that the myocardial damage caused by ICD shock may have a significant adverse influence on a poorly functioning myocardium. Therefore, low-energy ICD shock, enabled by lower defibrillation

Received November 25, 2015; revised manuscript received January 11, 2016; accepted January 21, 2016; released online February 17, 2016 Time for primary review: 11 days

Division of Cardiovascular Medicine, Toho University Ohashi Medical Center, Tokyo (M.N., Y.E., R.I., Y.T., M.A., N.S., T.T., Y.N., H.H., N.I., S.K., K.N., T. Sakata, K.S.); Biomedical Information Technology Lab, University of Aizu, Aizu-Wakamatsu (X.Z.); Boston Scientific Japan, Tokyo (Y.O., H.T.); Division of Cardiovascular Medicine, Saiseikai Yokohama Eastern Hospital, Yokohama (T. Sakai); and Second Department of Internal Medicine, School of Medicine, University of Occupational and Environmental Health, Kitakyushu (H.A.), Japan

The first two authors contributed equally to this work (M.N., X.Z.).

Mailing address: Mahito Noro, MD, PhD, Division of Cardiovascular Medicine, Toho University Ohashi Medical Center, 2-17-6 Ohashi, Meguro-ku, Tokyo 153-8515, Japan. E-mail: noro@oha.toho-u.ac.jp

ISSN-1346-9843 doi:10.1253/circj.CJ-15-1258

All rights are reserved to the Japanese Circulation Society. For permissions, please e-mail: cj@j-circ.or.jp 
$\mathbf{A}$

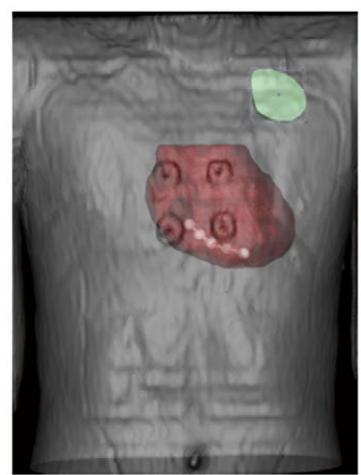

B

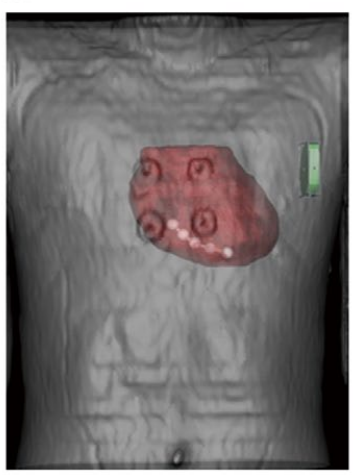

C

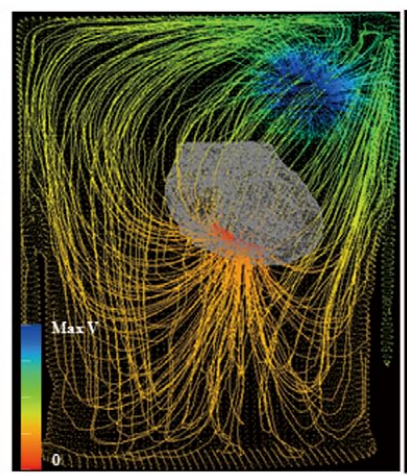

F

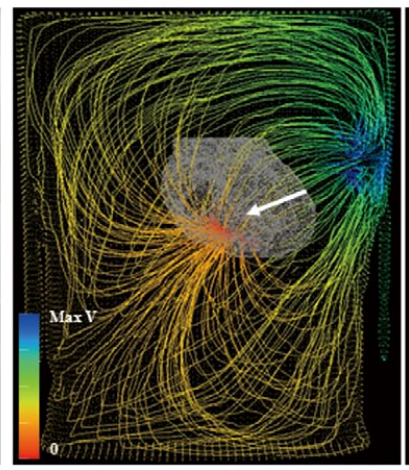

D

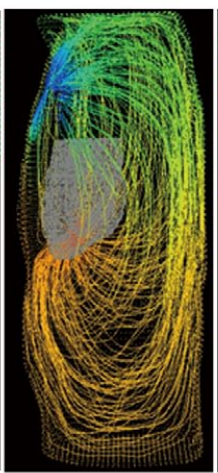

G

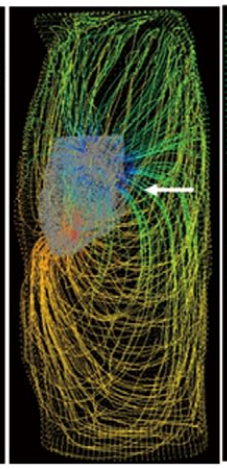

$\mathbf{E}$

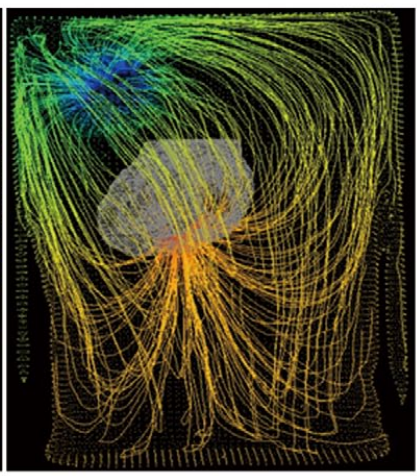

$\mathbf{H}$

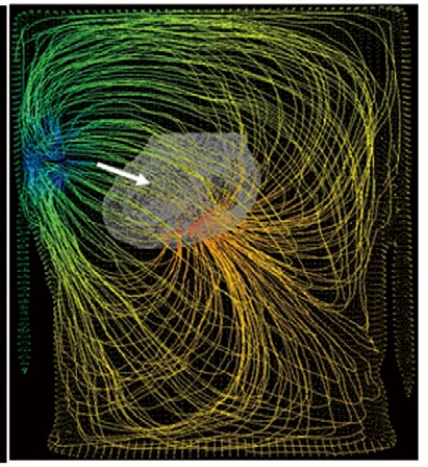

Figure 1. Simulations of electrical conduction pattern through the body during implantable cardioverter defibrillator (ICD) shock vs. site of ICD generator implantation: (A) left anterior chest; (B) axillary implantation. (C-H) Front, left lateral, and posterior views of the simulated electric field lines for (C-E) ICD generator implantation site (A); and (F-H) ICD generator implantation site (B).

threshold (DFT), may reduce the myocardial damage and thus improve the prognosis.

In addition, the electrical conduction is determined by the spatial distribution of the electrical conductivity of the tissue, and usually is not directly propagated in the human body. Because the DFT is related to the distribution of the defibrillation currents, the study of DFT should be based on the electrical conduction of defibrillation shocks.

In our previous study, pacemaker pulse generators were implanted in the left axilla and the safety, effectiveness, comfort, and cosmetic outcome of that implantation site were confirmed. ${ }^{14}$ In this study, the left axillary chest was investigated as an alternate ICD generator implantation site theoretically and experimentally, and effective defibrillation with less myocardial damage was confirmed on computer simulation.

\section{Methods}

\section{Computer Simulation}

In order to theoretically analyze the conduction of defibrillation shocks, finite element modeling of the defibrillation was conducted using SCIRun and a dataset developed by an Open Source software project of the SCI Institute's NIH/NIGMS CIBC Center. ${ }^{15}$ The SCIRun database includes a torso model built from cardiac gated magnetic resonance imaging (MRI) of a healthy, 19-year-old volunteer. ${ }^{16}$ The spatial interval between neighboring images was $5 \mathrm{~mm}$, and every image has a dimen- sion of $256 \times 256$. The boundary nodes, surface, volume, and meshes were obtained to construct a geometrical model of the human thorax. The torso models were piecewise made heterogeneous by setting different electrical conductivities for the different tissues: bowel gas, 0.002; connective tissue, 0.220; liver, 0.150; kidney, 0.070; skeletal muscle, 0.250; fat, 0.050; bone, 0.006; lung, 0.067; blood, 0.700; and myocardium, 0.250 siemens $/ \mathrm{m} .{ }^{17}$ By modifying the torso model, the 2 models in Figures 1A,B were used to simulate the potential distribution and spatial voltage gradients during the ICD defibrillation when the ICD generator was placed in the left anterior chest and left axilla, respectively. The defibrillation energy was calculated using $\mathrm{E}=1 / 2 \times \mathrm{C} \times \mathrm{V}^{2},{ }^{15}$ where $\mathrm{C}=130 \mu \mathrm{F}$, and $\mathrm{V}$ is the defibrillation voltage when a critical mass criterion is reached. Also note that a simulation was performed with a monophasic defibrillation waveform in order to reproduce the defibrillation current conduction and avoid the computation complexity in the simulation of a biphasic defibrillation waveform. The critical mass criterion was defined as $90 \%$ of the myocardium with a voltage gradient $>5 \mathrm{~V} / \mathrm{cm}$, which was the defibrillation gradient threshold. ${ }^{15,18}$ Further, we calculated the ventricular mass with a voltage gradient $>30 \mathrm{~V} / \mathrm{cm}$, which was the damage gradient threshold as an index to predict any possible myocardial damage..$^{15,19}$ In the Noro et al study, the Utah model was used for the computer simulation of subcutaneous ICD. ${ }^{20}$ Given that the maximum output of ICD devices is $36 \mathrm{~J}$ for Biotronik, $35 \mathrm{~J}$ for Boston Scientific, $35 \mathrm{~J}$ for Medtronic, $35 \mathrm{~J}$ 


\begin{tabular}{|c|c|c|c|c|c|c|c|c|c|}
\hline $\begin{array}{l}\text { Patient } \\
\text { ID. no. }\end{array}$ & Disease & Gender & $\begin{array}{c}\text { Age } \\
\text { (yeas) }\end{array}$ & DFT $\leq 5$ & EF (\%) & BMI (\%) & Drug & Device & Manufacturer \\
\hline 1 & OMI & $\mathrm{F}$ & 70 & Y & 43 & 18.1 & Sota BB & ICD & BSJ \\
\hline 2 & OMI & M & 78 & Y & 49 & 20.5 & Amio & ICD & BSJ \\
\hline 3 & OMI & M & 82 & Y & 43 & 22.5 & Sota & ICD & SJM \\
\hline 4 & OMI & M & 76 & $\mathrm{~N}$ & 38 & 27.3 & Amio, BB & ICD & Med \\
\hline 5 & OMI & M & 58 & $\mathrm{~N}$ & 39 & 27.3 & Amio & ICD & Bio \\
\hline 6 & OMI & M & 67 & Y & 54 & 28.5 & None & ICD & Med \\
\hline 7 & IVF & M & 22 & Y & 71 & 20.0 & None & ICD & Sorin \\
\hline 8 & IVF & M & 57 & Y & 69 & 20.3 & Bepri & ICD & SJM \\
\hline 9 & IVF & M & 79 & $Y$ & 69 & 22.9 & Bepri, BB & ICD & Sorin \\
\hline 10 & IVT & $\mathrm{F}$ & 76 & Y & 39 & 14.5 & Bepri & ICD & SJM \\
\hline 11 & IVT & M & 66 & $Y$ & 53 & 20.0 & Sata, Fleca & ICD & SJM \\
\hline 12 & IVT & $\mathrm{F}$ & 38 & Y & 73 & 21.6 & BB & ICD & BSJ \\
\hline 13 & IVT & M & 65 & Y & 63 & 27.5 & Amio & ICD & Sorin \\
\hline 14 & IVT & $\mathrm{F}$ & 64 & $Y$ & 68 & 27.6 & None & ICD & Med \\
\hline 15 & IVT & M & 65 & $Y$ & 69 & 28.2 & BB & $I C D$ & Sorin \\
\hline 16 & DCM & M & 75 & $Y$ & 20 & 20.8 & BB & CRT-D & SJM \\
\hline 17 & $\mathrm{DCM}$ & $\mathrm{F}$ & 77 & $Y$ & 32 & 23.0 & BB & CRT-D & BSJ \\
\hline 18 & DCM & M & 57 & $\mathrm{Y}$ & 28 & 23.5 & BB & CRT-D & BSJ \\
\hline 19 & DCM & $\mathrm{F}$ & 57 & $\mathrm{~N}$ & 33 & 28.9 & Amio, BB & ICD & Med \\
\hline 20 & $\mathrm{DCM}$ & M & 73 & $Y$ & 15 & 19.7 & Amio BB, Mexi & CRT-D & BSJ \\
\hline 21 & $\mathrm{BrS}$ & $\mathrm{F}$ & 20 & $\mathrm{Y}$ & 47 & 21.2 & None & ICD & SJM \\
\hline 22 & $\mathrm{BrS}$ & M & 50 & $\mathrm{Y}$ & 75 & 19.9 & Bepri, Quni & ICD & SJM \\
\hline 23 & BrS & M & 55 & Y & 64 & 20.5 & None & ICD & SJM \\
\hline 24 & Salcoidosis & $\mathrm{F}$ & 33 & $Y$ & 36 & 14.2 & Bepri & CRT-D & SJM \\
\hline 25 & Salcoidosis & M & 47 & $\mathrm{~N}$ & 28 & 19.6 & BB & ICD & BSJ \\
\hline 26 & J wave & $\mathrm{F}$ & 28 & Y & 77 & 20.5 & Amio & ICD & Bio \\
\hline 27 & Long QT & M & 67 & $Y$ & 82 & 25.2 & Mexi & ICD & BSJ \\
\hline 28 & VHD & M & 81 & $Y$ & 38 & 22.6 & Sota, BB & $I C D$ & Med \\
\hline 29 & VHD & M & 67 & $Y$ & 18 & 23.1 & BB & CRT-D & BSJ \\
\hline 30 & $\mathrm{HCM}$ & $\mathrm{F}$ & 46 & $Y$ & 68 & 19.2 & Amio & ICD & Sorin \\
\hline
\end{tabular}

Amio, amiodarone; BB, $\beta$-blocker; Bepri, bepridile; Bio, Biotronik; BMI, body mass index; BrS, Brugada syndrome; BSJ, Boston Scientific Japan; CRT-D, cardiac resynchronization therapy defibrillator; DCM, dilated cardiomyopathy; DFT, defibrillation threshold; EF, ejection fraction; Fleca, flecainide; HCM, hypertrophic cardiomyopathy; ICD, implantable cardioverter defibrillator; IVF, idiopathic ventricular fibrillation; IVT, idiopathic ventricular tachycardia; Med, Medtronic; Mexi, mexiletine; OMI, old myocardial infarction; Quni, quinidine; SJM, St. Jude Medical; Sorin, Sorin Group; Sota, sotacole; VHD, valvular heart disease; VSA, vasospastic angina.

for Sorin, and $40 \mathrm{~J}$ for St. Jude Medical, respectively, we estimated myocardial damage up to an ICD output of $40 \mathrm{~J}$ in the simulations.

\section{Clinical Experience}

During September 2012 and December 2014, we recruited 30 patients, who received a new ICD or cardiac resynchronization therapy defibrillator (CRT-D), with the ICD generator implanted in the left axilla. Table 1 lists disease, gender, age, DFT, ejection fraction (EF), body mass index (BMI), and the makers of the implanted devices. The locations of the ICD leads in the right ventricle (RV) and ICD generator are shown in Figures 2A-C.

To implant the ICD generator in the left axilla, we used a left axillary vein direct puncture method and a left axillary pocket. To implant a CRT-D, the lead was inserted into the subclavian vein as usual using a Penrose drain to avoid dislodgement of the left ventricle (LV) lead. After the implantation, no special postoperative instructions were given to the subjects with an ICD or CRT-D implanted in the left axilla. The details of the procedure for the left axillary ICD implantation were similar to those for the left axillary pacemaker implantation. ${ }^{14}$ Except for the subclavian vein puncture method, the method of implantation of the CRT-D generator in the left axilla was the same as that of the ICD generator.

Furthermore, during the same period, we performed new ICD implantations in the left anterior chest in 40 patients (Table 2). The locations of the ICD leads in the RV and the ICD generator are shown in Figures 2A,D,E.

During ICD testing, the initial ICD output was set to $5 \mathrm{~J}$. If the ventricular fibrillation (VF) could not be terminated using an output configuration of $5 \mathrm{~J}$, ICD shocks with higher energy output were used to end VF. All defibrillations were performed using a single coil configuration (RV coil to pulse generator) regardless of the type of actual lead (single or dual coil).

The devices used in the left axillary group were made by Biotronik $(n=2)$, Boston Scientific $(n=9)$, Medtronic $(n=5)$, Sorin ( $\mathrm{n}=5$ ), and St. Jude Medical ( $\mathrm{n}=9$ ), respectively. Twentyfour of these devices were ICD and the other 6 were CRT-D (Table 1). In the left anterior chest group, the devices were made by Biotronik ( $\mathrm{n}=1)$, Boston Scientific $(\mathrm{n}=15)$, Medtronic $(n=11)$, Sorin $(n=4)$, and St. Jude Medical $(n=9)$, respectively. Twenty-four of these devices were ICD, and the other 16 were CRT-D (Table 2). For ICD made by Medtronic and St. Jude 
B

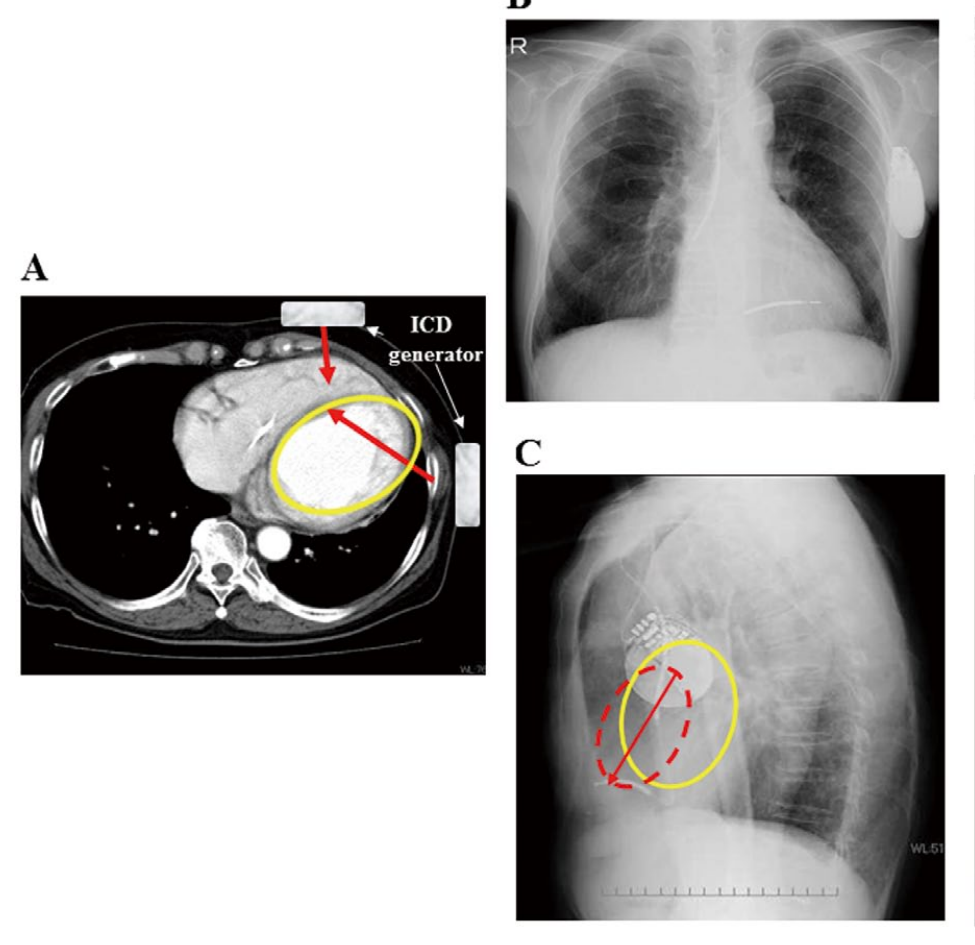

D

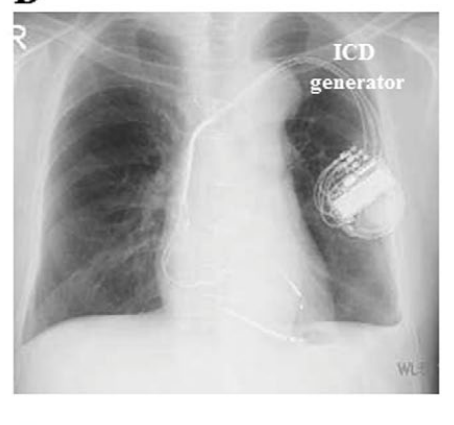

$\mathbf{E}$

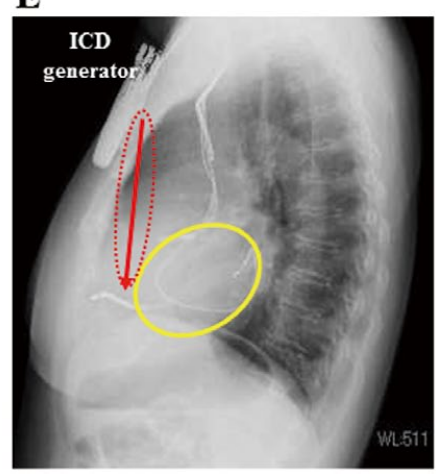

Figure 2. (A) Thoracic computed tomography slice of the heart. (B-E) Front and lateral views on chest radiograph with implantable cardioverter defibrillator (ICD) generator implantation in the (B,C) left axilla and (D,E) left axilla. Red arrows, most direct conduction route of the defibrillation current; yellow circle, left ventricle; rectangle, ICD generator; red dashed circle, range of defibrillation current in a homogeneous medium.

Medical, the actual output of the ICD is equivalent to the configured output because the delivered energy can be set in the ICD. In contrast, the actual output is less than the configured output in the Boston Scientific, Sorin, and Biotronik ICD because only the stored energy can be set. As a result, for Boston Scientific, Sorin, and Biotronik ICD, the output of DFT in the DFT testing was $<5 \mathrm{~J}$ if VF were terminated by a shock of $5 \mathrm{~J}$. In view of the variations in the different output configuration methods for ICD according to the manufacturer, the primary endpoint of the DFT testing was the termination of VF by a defibrillation shock output $\leq 5 \mathrm{~J}$.

This study was approved by the ethics committee of Toho University Ohashi Medical Center, Tokyo and was in compliance with the principles of the declaration of Helsinki. The procedure for the pulse generator implantation in the left axillary region was explained in detail to the patients, and was performed only in patients who agreed and were willing to undergo this procedure. Informed consent for implantation, and for the use and publication of the data were obtained from the patients before ICD implantation.

\section{Statistical Analysis}

Chi-squared test was used for comparison of the 2 groups regarding basic disease, gender, EF, BMI, medication, DFT, and device manufacturer. T-test was used for the analysis of age. Multiple logistic regression analysis was also used to investigate influence of implantation site, basic disease, gender, age, EF, BMI, medication, ICD type (ICD or CRT-D), and manufacturer on DFT.

\section{Results \\ Computer Simulation of ICD Defibrillation}

The simulated defibrillation electric field lines in the front, left lateral, and posterior views based on the 2 models are shown in Figures $\mathbf{1 C}-\mathbf{E}$ and Figures $\mathbf{1 F}-\mathbf{H}$, respectively. Because the lungs and bones have a lower electrical conductivity, ${ }^{17}$ regardless of the implantation site of the ICD generator, the simulated electric field lines generally circumvent the lungs and spine. When the ICD generator was placed in the left anterior chest, the electric field lines mainly traveled in the posterior and precordial regions in the upward and downward directions, respectively (Figure 1C). The upward electric field lines reached the heart via 2 pathways: 1 ran from the bottom of the heart's posterior wall to the heart, and the other ran down to the back of the abdomen and then to the front of the abdomen, and finally ended at the heart from the bottom of the heart's anterior wall. When the ICD generator was placed in the left axilla, the simulated electric field lines mainly traveled in 3 directions (Figure 1F, middle row). One ran upward; 1 downward; and 1 across the precordial and posterior regions to reach the heart.

Figures 3A-F uses the isopotential surfaces to illustrate the simulated defibrillation potential distribution throughout the body during ICD shocks when the ICD generator is placed in the left anterior chest and axilla, respectively. The isopotential surfaces were perpendicular to the electric field lines. The isopotential surfaces in the mediastinum of the anterior region were denser at the anterolateral wall of the RV when the ICD was implanted in the left anterior chest (Figures 3A-C). Never- 


\begin{tabular}{|c|c|c|c|c|c|c|c|c|c|}
\hline $\begin{array}{l}\text { Patient } \\
\text { ID no. }\end{array}$ & Disease & Gender & $\begin{array}{c}\text { Age } \\
\text { (yeas) }\end{array}$ & $\mathrm{DFT} \leq 5 \mathrm{~J}$ & $E F(\%)$ & BMI (\%) & Drug & Device & Manufacturer \\
\hline 1 & OMI & $\mathrm{M}$ & 76 & $\mathrm{Y}$ & 49 & 19.2 & Amio, BB & ICD & BSJ \\
\hline 2 & OMI & $\mathrm{M}$ & 77 & $\mathrm{Y}$ & 17 & 23.3 & Amio, BB & ICD & BSJ \\
\hline 3 & OMI & $M$ & 81 & $Y$ & 69 & 25.8 & Amio, BB & ICD & BSJ \\
\hline 4 & OMI & M & 70 & $\mathrm{Y}$ & 21 & 21.9 & BB & CRT-D & Med \\
\hline 5 & OMI & M & 73 & $\mathrm{Y}$ & 10 & 19.3 & Bepri, BB & CRT-D & Med \\
\hline 6 & $\mathrm{OMI}$ & $M$ & 66 & $\mathrm{Y}$ & 21 & 20.0 & Amio, BB & CRT-D & Med \\
\hline 7 & OMI & $\mathrm{M}$ & 69 & $N$ & 50 & 20.1 & Sota, BB & ICD & Sorin \\
\hline 8 & $\mathrm{OMI}$ & $\mathrm{F}$ & 55 & $\mathrm{~N}$ & 60 & 24.2 & BB & CRT-D & SJM \\
\hline 9 & OMI & $\mathrm{M}$ & 69 & $\mathrm{~N}$ & 35 & 22.4 & BB & CRT-D & BSJ \\
\hline 10 & $\mathrm{OMI}$ & $M$ & 72 & $\mathrm{~N}$ & 25 & 24.6 & Amio, BB & CRT-D & SJM \\
\hline 11 & OMI & $M$ & 83 & $\mathrm{~N}$ & 39 & 22.3 & Amio & ICD & SJM \\
\hline 12 & $\mathrm{OMI}$ & $M$ & 82 & $\mathrm{~N}$ & 23 & 23.6 & Amio & ICD & Sorin \\
\hline 13 & OMI & $M$ & 75 & $\mathrm{~N}$ & 40 & 24.6 & Sota & CRT-D & Sorin \\
\hline 14 & $\mathrm{OMI}$ & $M$ & 70 & $\mathrm{~N}$ & 23 & 20.0 & Amio, BB & CRT-D & BSJ \\
\hline 15 & $\mathrm{OMI}$ & $M$ & 72 & $\mathrm{~N}$ & 28 & 22.0 & Amio & ICD & BSJ \\
\hline 16 & OMI & $M$ & 75 & $\mathrm{~N}$ & 33 & 18.9 & Amio, BB, Mexi & ICD & Med \\
\hline 17 & OMI & $\mathrm{F}$ & 75 & $\mathrm{~N}$ & 24 & 17.5 & Amio, BB & ICD & Med \\
\hline 18 & OMI & $M$ & 65 & $\mathrm{~N}$ & 33 & 22.9 & None & CRT-D & SJM \\
\hline 19 & $\mathrm{HCM}$ & $M$ & 60 & $\mathrm{~N}$ & 27 & 24.1 & None & CRT-D & Med \\
\hline 20 & $\mathrm{HCM}$ & M & 66 & $\mathrm{~N}$ & 33 & 27.4 & Amio, BB & ICD & Med \\
\hline 21 & $\mathrm{HCM}$ & $\mathrm{F}$ & 51 & $\mathrm{~N}$ & 55 & 18.6 & Amio, BB & CRT-D & Med \\
\hline 22 & $\mathrm{HCM}$ & M & 70 & $\mathrm{~N}$ & 41 & 24.0 & BB & CRT-D & SJM \\
\hline 23 & $\mathrm{HCM}$ & $M$ & 44 & $\mathrm{~N}$ & 71 & 34.9 & BB & ICD & $\mathrm{BIO}$ \\
\hline 24 & IVF & $M$ & 33 & $\mathrm{~N}$ & 72 & 23.0 & None & CRT-D & Sorin \\
\hline 25 & IVF & $M$ & 79 & $\mathrm{~N}$ & 69 & 21.3 & Amio & CRT-D & Med \\
\hline 26 & IVT & $M$ & 78 & $N$ & 62 & 20.7 & BB & ICD & SJM \\
\hline 27 & IVT & $M$ & 67 & $\mathrm{~N}$ & 20 & 17.6 & Amio & $I C D$ & SJM \\
\hline 28 & BrS & $M$ & 27 & $Y$ & 55 & 22.1 & None & ICD & BSJ \\
\hline 29 & $\mathrm{BrS}$ & $M$ & 58 & $\mathrm{~N}$ & 72 & 25.9 & None & ICD & BSJ \\
\hline 30 & $\mathrm{BrS}$ & $M$ & 43 & $N$ & 65 & 19.4 & None & ICD & BSJ \\
\hline 31 & DCM & $M$ & 48 & $\mathrm{~N}$ & 13 & 30.3 & None & CRT-D & BSJ \\
\hline 32 & $\mathrm{DCM}$ & $\mathrm{F}$ & 68 & $Y$ & 37 & 22.0 & BB & ICD & Med \\
\hline 33 & Salcoidosis & $\mathrm{F}$ & 71 & $Y$ & 24 & 20.9 & None & ICD & SJM \\
\hline 34 & Salcoidosis & $M$ & 46 & $\mathrm{~N}$ & 19 & 19.5 & Sota & ICD & SJM \\
\hline 35 & Salcoidosis & $\mathrm{F}$ & 79 & $\mathrm{~N}$ & 28 & 21.3 & BB & CRT-D & BSJ \\
\hline 36 & Amiroidosis & $M$ & 77 & $N$ & 66 & 23.0 & None & ICD & BSJ \\
\hline 37 & J wave & $M$ & 44 & $\mathrm{~N}$ & 58 & 19.3 & None & ICD & BSJ \\
\hline 38 & Long QT & $M$ & 68 & $Y$ & 55 & 25.2 & Mexi & $I C D$ & BSJ \\
\hline 39 & VHD & $M$ & 77 & $\mathrm{~N}$ & 40 & 21.8 & Amio & ICD & Med \\
\hline 40 & VSA & $M$ & 43 & $Y$ & 62 & 18.0 & None & ICD & BSJ \\
\hline
\end{tabular}

Abbreviations as in Table 1.

theless, the isopotential surfaces of the left axillary implantation were denser from the lateral wall of the ICD generator to the LV (Figures 3D-F), and denser in the LV (Figures 3I,J) compared with those of the left anterior implantation (Figures $3 \mathbf{G}, \mathbf{H}$ ). The simulated isopotential surfaces in the 2 implantation sites had similar densities in the right apical region.

Neither the LV nor the RV masses met the critical mass criterion for the whole ventricle. In Figure $3 \mathbf{K}$, the red regions have a voltage gradient below the defibrillation gradient threshold, which occurred mainly in the LV in the left anterior chest implantation. In contrast, in the left axillary implantation, voltage gradient below the defibrillation gradient threshold occurred close to the ventricular base and extended to the atria (Figure 3L).
In the anterior chest implantation, ventricular mass with voltage gradient above the defibrillation gradient threshold was larger in the RV and smaller in the LV (Figure 4A). In contrast, in the left axillary implantation, voltage gradient above the defibrillation gradient threshold had approximately the same distribution in the RV, LV, and the whole heart (Figure 4B).

The DFT of the left anterior chest implantation were 9.4 J $(380 \mathrm{~V}), 12.0 \mathrm{~J}(430 \mathrm{~V})$, and $4.7 \mathrm{~J}(270 \mathrm{~V})$ for the whole heart, LV, and RV, respectively (Figures 4A,C). The DFT of the axillary implantation were $6.2 \mathrm{~J}(310 \mathrm{~V}), 6.4 \mathrm{~J}(315 \mathrm{~V})$, and $6.0 \mathrm{~J}(305 \mathrm{~V})$ for the whole heart, $\mathrm{LV}$, and $\mathrm{RV}$, respectively, indicating a lower DFT (Figures 4B,C). 

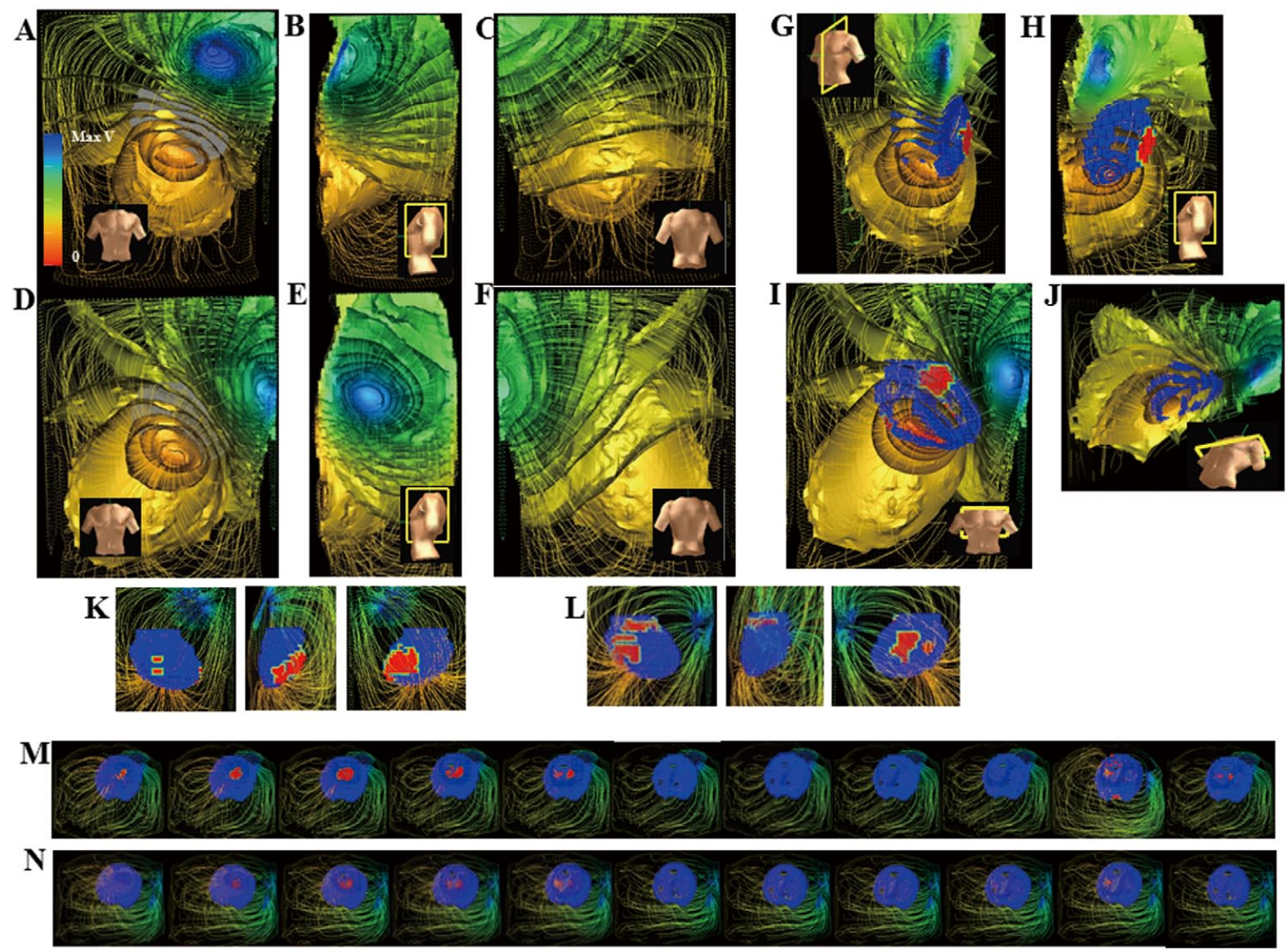

Figure 3. Simulated distribution of the defibrillation voltage within the body for (A-C) left anterior chest implantation and (D-F) left axillary implantation of implantable cardioverter defibrillator (ICD) generator. (G,H) Distribution of the voltage in the sagittal plane with the heart in the $(\mathbf{G})$ right anterior oblique and $(\mathbf{H})$ left lateral views for left anterior chest ICD generator location. (I,J) Distribution of the voltage in (I) the frontal plane and ( $\mathbf{J}$ ) an inclined plane for axillary ICD generator location. Blue, heart; red, voltage spatial difference lower than the defibrillation gradient threshold. (K,L) Distribution of myocardium with (red) voltage gradient lower than the defibrillation gradient threshold (also shown in red in $\mathbf{G}-\mathbf{J}$ ) in the (K) left anterior chest and (L) left axillary implantations. (M,N) Distribution of myocardium with voltage gradient (red) above the damage gradient threshold in the (M) left anterior chest and $(\mathrm{N})$ left upper axillary implantations. Slices are from the apex to the base of the ventricle.

\section{Potential for Tissue Damage}

The corresponding myocardial damage areas in the simulations were localized in the RV apex region surrounding the shock coil in the left anterior chest and left axillary implantations (Figures 3M,N), where the slices were from the ventricular apex to the base. In the simulation, when the critical mass criterion was reached for the left anterior chest implantation, the proportion of ventricular mass above the damage gradient threshold was $4.2 \%, 0 \%$, and $6.8 \%$ for the whole heart, $\mathrm{LV}$, and RV, respectively (Figure 4D). The corresponding values were reduced to $2.1 \%, 0 \%$, and $3.4 \%$ in the left axillary implantation (Figure 4E). Thus, compared with the simulated left anterior chest implantation (Figure 4F), the potential myocardial damage was better controlled in the axillary implantation.

When the ICD output was set to $40 \mathrm{~J}$ (maximum output) in the simulation, the myocardial damage ratios in the whole heart, LV, and RV were $16.4 \%$ vs. $19.4 \%, 0 \%$ vs. $3.1 \%$, and $26.4 \%$ vs. $29.2 \%$ for the left anterior chest and left axillary implantations, respectively (Figures 4D,E). Furthermore, the LV damage region was focused in the apical area for the left axillary implantation when the shock energy of the simulation was set to $40 \mathrm{~J}$.

\section{Human Clinical Experience}

The VF in $26 / 30$ patients $(86.7 \%$ ) in the left axillary group was terminated by a defibrillation output configuration of $5 \mathrm{~J}$ (delivery or storage energy), but only $11 / 40$ patients (27.5\%) had DFT $\leq 5 \mathrm{~J}$ in the left anterior chest group (Tables 1,2). Significant difference was observed in the clinical DFT of the 2 groups, but there was no significant difference in gender, age, EF, or BMI (Table 3). On multiple logistic regression analysis, implantation site, EF, BMI, and medications had OR 25.8, 0.28, 0.27, and 0.34 , respectively (Table 4 ). Thus, the left axillary implantation may significantly reduce DFT, but DFT may increase with reduction in $\mathrm{EF}$, increasing $\mathrm{BMI}$, and prescription of amiodarone.

After implantation, patients with ICD visit the outpatient clinic every 4 months. The patients usually had discomfort immediately after the implantation, but this gradually disappeared within 4 months. We checked chest radiograph, sens- 
A The left anterior chest

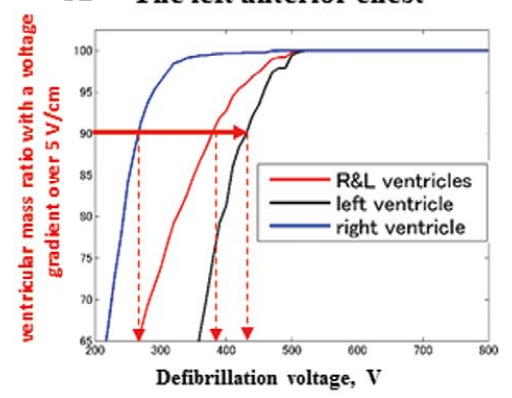

D The left anterior chest

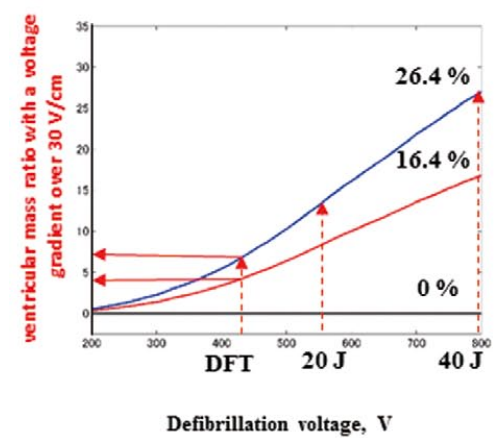

B The left axilla

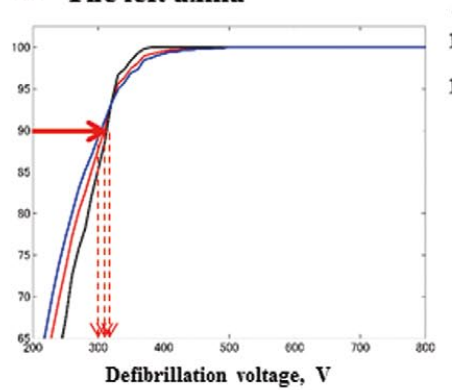

E The left axilla

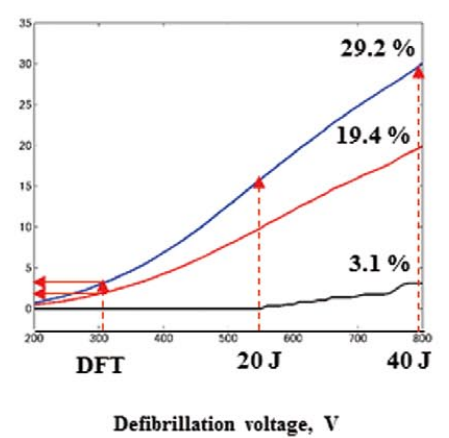

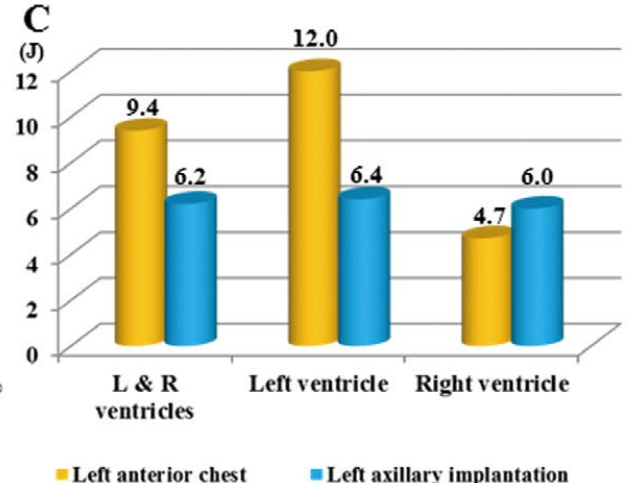

$\mathbf{F}$

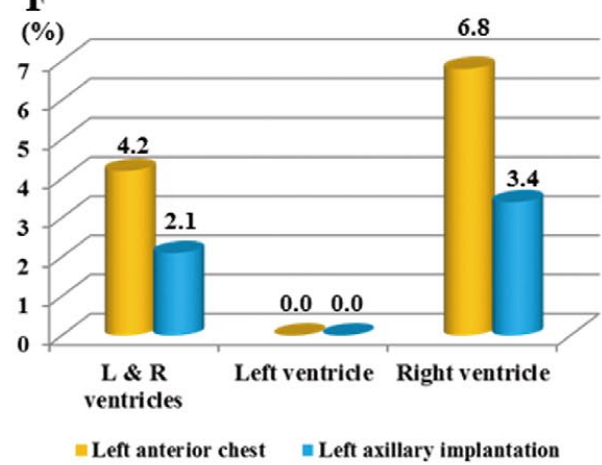

Figure 4. Relationship between implantable cardioverter defibrillator (ICD) generator implantation site, defibrillation threshold (DFT), and myocardial damage. (A,B) Relationship between ventricular mass with voltage gradient above the defibrillation gradient threshold and defibrillation voltage. (C) DFT when both ventricles, the left, or right ventricle satisfied the critical mass criterion in the left anterior chest and left axillary implantation sites, respectively. (D,E) Relationship between ICD generator defibrillation output and proportion of ventricular mass with voltage gradient above the damage gradient threshold. (F) Ventricular mass with myocardial damage vs defibrillation output equal to the DFT of the whole heart.

\begin{tabular}{|c|c|c|c|}
\hline & $\begin{array}{l}\text { Left axillary } \\
\text { group }\end{array}$ & $\begin{array}{l}\text { Left anterior } \\
\text { chest group }\end{array}$ & P-value \\
\hline Male & $34(85)$ & $20(67)$ & 0.07 \\
\hline Age (years) & $65.0 \pm 14.3$ & $59.9 \pm 17.6$ & 0.18 \\
\hline$E F<40 \%$ & $21(52.5)$ & $12(40.0)$ & 0.30 \\
\hline $\mathrm{BMI} \leq 40 \%$ & $10(25.0)$ & 7 (23.3) & 0.64 \\
\hline DFT & & & $<0.05^{*}$ \\
\hline$\leq 5 \mathrm{~J}$ & $11(27.5)$ & $26(86.7)$ & \\
\hline$>5 \mathrm{~J}$ & $29(72.5)$ & 4 (13.3) & \\
\hline
\end{tabular}

${ }^{*} \mathrm{P}<0.05$. Data given as mean $\pm \mathrm{SD}$ or $\mathrm{n}(\%)$. Abbreviations as in Table 1.

ing, impedance, and so on, on follow-up visits but did not note any migration, infection or complications. Thus, no generator replacement was performed. Furthermore, because the axilla has a relatively large and deep anatomical structure and is filled with fat tissues, the ICD/CRT-D generators implanted in the left axilla were hidden in the axilla and caused no skin trouble.

\section{Discussion}

With regard to simulation results, (1) the conducted defibrillation current avoided the lungs and spine due to their high resistance; (2) the DFT in the left axillary implantation was lower than that in the left anterior chest implantation; and (3) the proportion of ventricular mass above the damage gradient threshold was lower when the ICD was implanted in the left axilla.

When the ICD generator was placed in the left anterior chest, the downward electric field lines traveled in 2 ways (Figure 1C). The main route was from the anterior and posterior mediastinum to the heart; the other came from the chest and back and went up from the abdomen to the heart via the posterior and anterior surfaces of the diaphragm.

If the electrical conduction pattern of the left anterior chest implantation (Figure 1C) turned clockwise, it would have been similar to that of the left axillary implantation (Figure 1F). Thus, ICD shocks were also conducted across the anterior and posterior regions.

Therefore, the electrical conduction in different ICD generator implantation sites may vary as to whether the electric field lines travel to the anterior or posterior regions in the bottom of the heart. It is hypothesized that the denser the electric field lines across the posterior region in the bottom of the 


\begin{tabular}{|c|c|c|c|}
\hline Index & $\beta(95 \% \mathrm{Cl})$ & OR & P-value \\
\hline Implantation site & 3.25 (1.57 to 4.94$)$ & 25.8 & 0.00 \\
\hline Disease & $0.11(-0.24$ to 0.47$)$ & 1.12 & 0.54 \\
\hline Gender & $-0.35(-1.96$ to 1.26$)$ & 0.70 & 0.67 \\
\hline Age & $0.68(-0.05$ to 1.40$)$ & 1.97 & 0.07 \\
\hline EF & $-1.27(-2.81$ to 0.28$)$ & 0.28 & 0.11 \\
\hline BMI & $-1.28(-2.65$ to 0.10$)$ & 0.27 & 0.07 \\
\hline Drug & $-1.07(-2.62$ to 0.47$)$ & 0.34 & 0.17 \\
\hline Device (CRT-D or ICD) & $-0.25(-1.81$ to 1.31$)$ & 0.78 & 0.75 \\
\hline Manufacturer & $-0.43(-1.10$ to 0.25$)$ & 0.65 & 0.21 \\
\hline
\end{tabular}

Abbreviations as in Table 1.

heart, the higher the defibrillation current conducted to the LV. In contrast, the denser the electric field lines across the anterior region in the bottom of the heart, the higher the defibrillation current conducted in the RV. That is to say, the defibrillation efficiency would be raised with higher ICD defibrillation current conducted to the LV from the posterior region of the diaphragm. ${ }^{18}$

The main difference between the defibrillation electrical conduction in the left anterior and axillary implantations was the existence of a conduction pathway intersecting the anterior and posterior regions in the left axillary implantation. The conduction mainly ran from the diaphragm to the bottom, and the corresponding defibrillation currents had a higher density in the anterior region than the posterior region. This is confirmed by the fact that the simulated isopotential surfaces (Figures 3A-F) had a higher density in the anterior region of the RV but a lower density in the LV for the left anterior chest implantation. In contrast, the simulated isopotential surfaces had a higher density in the LV in the left axillary implantation. This may have led to a higher defibrillation efficiency and lower DFT.

Therefore, on the simulation, a greater ICD energy output was required if the $\mathrm{LV}$ met the critical mass criterion in the left anterior chest implantation. This was proven experimentally because the LV DFT in the left anterior chest implantation was $12.0 \mathrm{~J}$, and $6.4 \mathrm{~J}$ in the axillary implantation. Furthermore, as well as the greater ICD defibrillation current conducted to the LV, the left axillary implant location may have had a more uniform defibrillation effect than the left anterior chest implant location because the LV and RV DFT were similar in the axillary implantation.

Moreover, when the ICD generator was implanted in the left anterior chest, the electric field lines pointed directly toward the RV through the front of the precordial region including the mediastinum, and traveled to the LV by passing around the back. If the conductivity in those regions was similar, the resistance was proportional to the length of the conduction route. Furthermore, the voltage between the ICD generator and lead was fixed. As a result, the defibrillation current in the LV decreased as the conduction route from the ICD generator to the LV became longer. In contrast, in the left axillary implantation, the electric field lines across the posterior regions had a shorter conduction pathway to the LV as compared with those via the precordial region. Therefore, the defibrillation currents were stronger via the posterior region to the LV. This may have been the reason for the improvement in the defibrillation effectiveness and lower DFT.

The myocardial damage ratio of the left axillary implantation, however, was larger for the maximum ICD output because the defibrillation currents are more efficiently conducted to the $\mathrm{LV}$ in this implantation. Nevertheless, the myocardial damage ratio of the $L V$ was $0 \%$ when the ICD output was $\leq 20 \mathrm{~J}$ for the 2 implantations in the simulation. Therefore, for cases of low DFT, it is feasible to set the initial DFT output as $20 \mathrm{~J}$, and set the maximum DFT output when the initial defibrillation fails. For cases of high DFT, in view of the increasing of DFT due to anti-arrhythmias drug treatment and the progress of cardiac disease, it is better to set the first defibrillation shock with the maximum output to achieve a successful defibrillation effect in the LV. This may not cause severe myocardial damage in the LV because the myocardial damage ratio was approximately $3 \%$ even for maximum ICD output in the simulation.

Although the association between ICD shock and late prognosis is controversial, ${ }^{3-8}$ myocardial damage caused by ICD shock has been studied using animal experiments and clinical troponin indices. In animal experiments, Xie et al found that the animal survival time and cardiac index decreased with increased shock energy. ${ }^{9}$ Yamaguchi et al observed that electrical shock produces myocardial injury in animals only when the myocardial perfusion is reduced. ${ }^{10}$ Hurst et al studied 49 patients and concluded that patients with a recent myocardial infarct and mean DFT $\geq 18 \mathrm{~J}$ tend to have myocardial injury with a rise in troponin. ${ }^{11}$ Davoodi et al found that DFT test shock might result in myocardial injury. ${ }^{12}$ In the Sham'a et al study, troponin elevation occurred in most of patients after inappropriate ICD shock because of lead fracture. ${ }^{13}$ Therefore, it was proven that ICD shock causes myocardial damage, and the severity of the myocardial damage is related to the defibrillation energy. It is not clear, however, whether the myocardial damage would improve over time or become chronic. Nevertheless, myocardial damage may be an important risk factor for prognosis. After all, the lesser the myocardial damage, the better the late prognosis might be, especially for patients with low cardiac function.

In human clinical experience, $86.7 \%$ of the patients with axillary implantation had DFT $\leq 5 \mathrm{~J}$, but only $27.5 \%$ in the control group had DFT $\leq 5 \mathrm{~J}$. Therefore, the left axillary implantation may improve the defibrillation effectiveness, with a significantly lower DFT with potentially less myocardial damage. Furthermore, lower DFT reduces energy consumption of the battery. Although this may help to reduce the size of the ICD battery, this may also reduce the defibrillation charge time due to the lower energy output, which may speed up defibrillation. As a result, in order to avoid inappropriate shock due to shorter charge time, the duration of the VT or VF sustenance in the ICD configuration may need to be increased for axillary implantation. ${ }^{21}$

The endpoint of the present DFT test was whether the VF was terminated by $5 \mathrm{~J}$. This endpoint was set because DFT 
testing after implantation may be necessary in patients with ICD generators implanted in the left axilla, in order to confirm the DFT and whether or not the ICD energy output is sufficient.

\section{Conclusion}

Implantation of ICD generator in the left axillary fossa instead of the traditional left anterior chest was studied. On computer simulation the defibrillation current was more efficiently conducted to the LV and therefore had a better defibrillation effect using this new implantation site. Clinical experience further validated this result. Hence, left axillary fossa implantation may lead to lower DFT compared with left anterior chest implantation, and therefore prevent myocardial damage due to ICD shock. Moreover, as well as reduction in myocardial damage, left axillary fossa implantation may also be useful for patients with high DFT.

\section{Study Limitations}

We conducted a computer simulation with the hypothesis of normal myocardium and heart structure. Conduction of the current may vary due to progression of cardiac failure and pulmonary congestion. In the clinical experience, pulse generators from different manufacturers were used, which might have different capacitances, and which may have led to variation in the DFT.

We used only one torso model in the study, but the simulation results would vary depending on use of torso models of patients with congestive heart failure, chronic obstructive pulmonary disease, pectus deformity, larger size, and so on. ${ }^{22}$ In this study, we mainly studied the conduction patterns of defibrillation shock for the anterior chest and left axillary implantations, but did not consider variation of electrical conductivity due to basic diseases.

In this study, implantation of ICD generator was carried out at the preferred site of each subject. Consequently, the patients were not randomly selected, and this study was not treated as a clinical study but rather clinical experience. Clinical study is planned in the future.

We considered only the monophasic defibrillation waveform in view of the lower mathematical complexity, because the simulation of biphasic defibrillation waveform involves the cardiac ion channels. We also expect to develop a diffusion-reaction model considering the cardiac ion channels, in order to simulate the defibrillation efficiency and lower cardiac damage of the biphasic defibrillation waveform.

\section{Acknowledgment}

The authors would like to thank Kazuya Murakami (Boston Scientific Japan) for detailed product knowledge relevant to supporting this work.

\section{Disclosures}

This work was supported in part by the Japan Society for the Promotion of Science under Grants-In-Aid for Scientific Research 25330339, and the National Institute of General Medical Sciences of the National Institutes of Health under grant number P41GM103545.

\section{References}

1. Satake H, Fukuda K, Sakata Y, Miyata S, Nakano M, Kondo M, et $\mathrm{al}$; CHART-2 Investigators. Current status of primary prevention of sudden cardiac death with implantable cardioverter defibrillator in patients with chronic heart failure: A report from the CHART-2 Study. Circ J 2015; 79: 381-390.

2. JCS Joint Working Group. Guidelines for non-pharmacotherapy of cardiac arrhythmias (JCS 2011): Digest version. Circ J 2013; 77: 249-274.

3. Poole JE, Johnson GW, Hellkamp AS, Anderson J, Callans DJ, Raitt $\mathrm{MH}$, et al. Prognostic importance of defibrillator shocks in patients with heart failure. N Engl J Med 2008; 359: 1009-1017.

4. Sweeney MO, Sherfesee L, DeGroot PJ, Wathen MS, Wilkoff BL. Differences in effects of electrical therapy type for ventricular arrhythmias on mortality in implantable cardioverter-defibrillator patients. Heart Rhythm 2010; 7: 353-360.

5. Swerdlow C, Ellenbogen KA, Klein GJ. Resolving the conflict: Implantable cardioverter-defibrillator shocks for ventricular tachyarrhythmias increase mortality. Heart Rhythm 2011; 9: 1328-1330.

6. Dorian P. Counterpoint: Implantable cardioverter-defibrillator shocks for ventricular tachyarrhythmias increase mortality. Heart Rhythm 2012; 9: 1158 .

7. Powell BD, Saxon LA, Boehmer JP, Day JD, Gilliam FR, Heidenreich PA, et al. Survival after shock therapy in implantable cardioverterdefibrillator and cardiac resynchronization therapy-defibrillator recipients according to rhythm shocked: The ALTITUDE Survival by Rhythm Study. J Am Coll Cardiol 2013; 62: 1674-1679.

8. Williams ES, Poole JE. Understanding implantable cardioverterdefibrillator shocks and mortality. J Am Coll Cardiol 2013; 62: $1680-1682$.

9. Xie J, Weil MH, Sun S, Tang W, Sato Y, Jin X, et al. High-energy defibrillation increases the severity of postresuscitation myocardial dysfunction. Circulation 1997; 96: 683-688.

10. Yamaguchi H, Weil MH, Tang W, Kamohara T, Jin X, Bisera J. Myocardial dysfunction after electrical defibrillation. Resuscitation 2002; 54: 289-296.

11. Hurst TM, Hinrichs M, Breidenbach C, Katz N, Waldecker B. Detection of myocardial injury during transvenous implantation of automatic cardioverter-defibrillators. J Am Coll Cardiol 1999; 34: 402-408.

12. Davoodi G, Mohammadi V, Shafiee A, Sadeghian S, VashghaniFarahani A, Yaminisharif A. Detection of myocardial injury due to defibrillation threshold checking after insertion of implantable cardioverter/defibrillators. Acta Cardiol 2013; 68: 167-172.

13. Sham'a RA, Nery P, Sadek M, Yung D, Redpath C, Perrin M, et al. Myocardial injury secondary to ICD shocks: Insights from patients with lead facture. Pacing Clin Electrophysiol 2014; 37: 237-241.

14. Noro M, Zhu X, Takagi T, Sahara N, Narabayashi Y, Hashimoto H, et al. Left axillary pacemaker generator implantation with a direct puncture of the left axillary vein. Pacing Clin Electrophysiol 2015; 38: $35-41$.

15. Jolley M, Stinstra J, Pieper S, MacLeod R, Brooks DH, Cecchin F, et al. A computer modeling tool for comparing novel ICD electrode orientations in children and adults. Heart Rhythm 2008; 5: 565-572.

16. Macleod RS, Johnson CR, Ershler PR, Harrison NE. Construction of a human torso model from magnetic resonance images for problems in computational electrocardiography. Univ Utah Comp Sci Tech Rep 1994; 17. http://www.sci.utah.edu/ macleod/papers/tr-mridoc. pdf (accessed September 13, 2015).

17. Miklavčič D, Pavšelj N, Hart FX. Electric properties of tissues. In: Akay M, editors. Wiley encyclopedia of biomedical engineering. New Jersey, USA, John Wiley \& Sons, 2006.

18. Zipes DP, Fischer J, King RM, Nicoll A, Jolly WW. Termination of ventricular fibrillation in dogs by depolarizing a critical amount of myocardium. Am J Cardiol 1975; 36: 37-44.

19. Tung L, Tovar O, Neunlist M, Jain SK, O'Neill RJ. Effects of strong electrical shock on cardiac muscle tissue. Ann NY Acad Sci 1994; 720: $160-175$.

20. Noro M, Zhu X, Enomoto Y, Asami M, Ishii R, Toyoda Y, et al. Efficacy and myocardial injury with subcutaneous implantable cardioverter defibrillators. Circ J 2016; 80: 85-93.

21. Noro M, Zhu X, Takagi T, Sahara N, Narabayashi Y, Hashimoto H, et al. Evaluation of defibrillation safety and shock reduction in ICD patients with increased time to detection: The randomized SANKS study. J Arrhythmia 2014; 31: 94-100.

22. Mizukami K, Yokoshiki H, Mitsuyama H, Watanabe M, Tenma T, Matsui Y, et al. Predictors of high defibrillation threshold in patients with implantable cardioverter-defibrillator using a transvenous dualcoil lead. Circ J 2014; 79: 77-84. 